\title{
Type A Aortic Dissection Presenting with Neurological Symptoms Mimicking Stroke and Intracranial Hemorrhage
}

\author{
Meng-Yu Wu 1,2 (D), Ying-Wei Tsai ${ }^{1,2}$, Ling-Chi Lee ${ }^{1,2}$, Yung-Chang Chien ${ }^{1,2}$, Yu-Long Chen ${ }^{1,2}$, \\ Yueh-Tseng Hou ${ }^{1,2}$ and Giou-Teng Yiang ${ }^{1,2, *}$ \\ 1 Department of Emergency Medicine, Taipei Tzu Chi Hospital, Buddhist Tzu Chi Medical Foundation, \\ New Taipei 231, Taiwan; skyshangrila@gmail.com (M.-Y.W.); fjm520@gmail.com (Y.-W.T.); \\ nakahikari@gmail.com (L.-C.L.); masonv1r@icloud.com (Y.-C.C.); yulong0129@gmail.com (Y.-L.C.); \\ brianann75@gmail.com (Y.-T.H.) \\ 2 Department of Emergency Medicine, School of Medicine, Tzu Chi University, Hualien 970, Taiwan \\ * Correspondence: gtyiang@gmail.com; Tel.: +886-2-6628-9779; Fax: +886-2-6628-9009
}

Received: 19 March 2018; Accepted: 23 March 2018; Published: 26 March 2018

\begin{abstract}
Acute aortic dissection is a rare but lethal cardiac disease involving the aorta, which presents with typical symptoms, including severe, sharp, or tearing acute onset of chest or back pain. Other unspecific symptoms, such as epigastric pain, nausea, and vomiting, may be also present. Neurologic symptoms induced by aortic dissection are also present in rare cases but often lead to delate diagnosis because they mimic stroke and intracranial hemorrhage. Therefore, it is important to understand acute aortic dissection-induced neurologic symptoms. Here, we present a case of a 60-year-old male who presented with an acute onset of progressive flaccid hemiplegia on the left side accompanied with slurred speech. Brain computed tomography showed widening of the cortical sulci and dilatation of the ventricles, without intracranial hemorrhage. When waiting for thrombolysis or thrombectomy intervention, acute chest pain was noted. After management, type A dissection from ascending aorta with extension to the right external iliac artery was noted in computed tomography angiography, which also involved the brachiocephalic trunk, left common carotid artery, celiac trunk, superior mesenteric artery, and right renal artery. This paper describes the clinical features of type A dissection, especially the neurological symptoms, and highlights the importance of early diagnosis and timely intervention for type A dissection patients.
\end{abstract}

Keywords: acute aortic dissection; type A dissection; stroke; intracranial hemorrhage

\section{Introduction}

Acute aortic dissection is a rare but lethal cardiac disease involving the aorta, which incident rate is reported as being 11/100,000 individuals [1]. Its typical symptoms include the acute onset of severe chest or back pain, which can be severe, sharp, or tearing. Although tearing chest pain is a typical symptom, other unspecific symptoms may be present, such as epigastric pain, nausea, and vomiting. Neurologic symptoms are also present in rare cases and often lead to delate diagnosis because they mimic stroke and intracranial hemorrhage. Therefore, it is important to understand acute aortic dissection-induced neurologic symptoms.

Here, we present a case of a 60-year-old male who presented with an acute onset of progressive flaccid hemiplegia on the left side, accompanied with slurred speech. Brain computed tomography showed widening of the cortical sulci and dilatation of the ventricles, without intracranial hemorrhage. When waiting for thrombolysis or thrombectomy intervention, acute chest pain was 
noted. An emergency computed tomography angiography was done and revealed type A dissection from the ascending aorta with extension to the right external iliac artery, which also involved the brachiocephalic trunk, left common carotid artery, celiac trunk, superior mesenteric artery, and right renal artery. This paper describes the clinical features of type A dissection, especially the neurological symptoms, and highlights the importance of early diagnosis and timely intervention for type A dissection patients.

\section{Case Presentation Section}

A 60-year-old male presented with acute onset of progressive flaccid hemiplegia on the left side, accompanied with slurred speech. His past medical history revealed poor controlled hypertension for five years. There was no history of trauma. He denied any infection sign, such as fever, chills, cough, nausea, vomiting, and diarrhea. Chest pain or tightness were not reported. There was no neurologic symptom, such as eye deviation, nystagmus, facial paralysis, dysarthria, or saliva drooling. His vital sign revealed a temperature of $36.1^{\circ} \mathrm{C}$, heart rate of 70 beats $/ \mathrm{min}$, respiratory rate of $20 \mathrm{breaths} / \mathrm{min}$ and blood pressure 136/59 $\mathrm{mm} \mathrm{Hg}$. On physical examination, the muscle power of the left extremity decreased and was asymmetric (the muscle strength in the upper extremities was $1 / 5$, but that of the lower extremities was 1/5). The extraocular motion was not checked because the patient could not comply. Laboratory data were collected to rule out infection diseases; the results were: white cell count of $13,970 / \mu \mathrm{L}$ (segment form neutrophils $70.3 \%$, lymphocytes $21.8 \%$, eosinophils $1.1 \%$, and monocytes 6.4\%), hemoglobin $10.6 \mathrm{~g} / \mathrm{dL}$, platelet counts $180,000 / \mathrm{mL}$, creatinine $1.3 \mathrm{mg} / \mathrm{dL}$, sodium $137 \mathrm{mmol} / \mathrm{L}$, potassium $2.7 \mathrm{mmol} / \mathrm{L}$, glucose $144 \mathrm{mg} / \mathrm{dL}$, alanine aminotransferase $30 \mathrm{U} / \mathrm{L}$, Troponin I < $0.01 \mu \mathrm{g} / \mathrm{L}, \mathrm{PT}: 12.3 \mathrm{~s}$, APTT: $33.1 \mathrm{~s}$, and INR: 1.19. The electrocardiogram revealed a normal sinus rhythm and prolonged QT. The supine anteroposterior chest view showed widening of the mediastinum (Figure 1A). Brain computed tomography showed widening of the cortical sulci and dilatation of the ventricles, without intracranial hemorrhage (Figure 1B). According to the above clinical symptoms and images, a stroke was suspected initially. The neurologists were consulted for thrombolysis or thrombectomy.
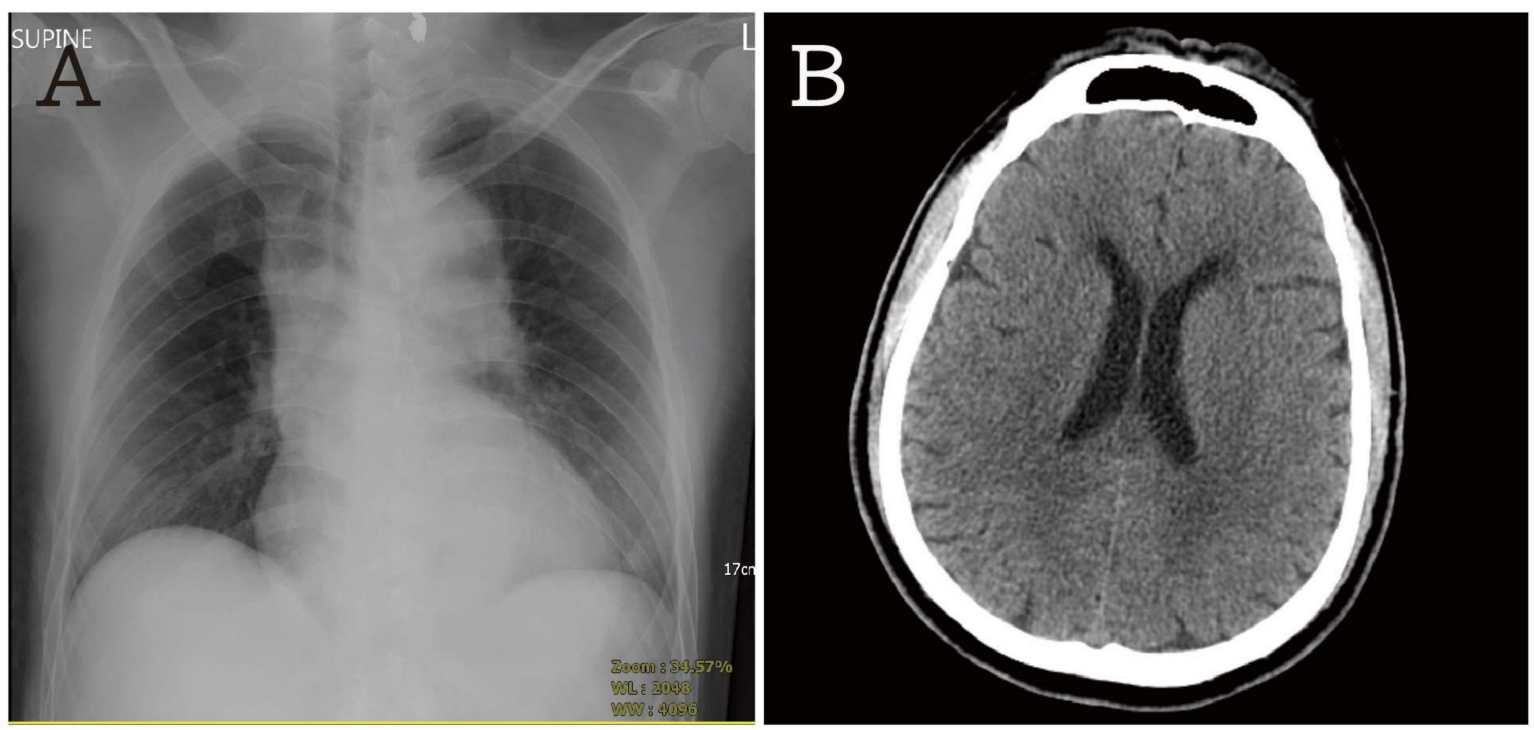

Figure 1. (A) The plain film revealed widening of the mediastinum. (B) There was no intracanal hemorrhage or mass effect in the brain computed tomography scan.

Acute chest pain was noted while waiting for the thrombolysis intervention. An emergency computed tomography angiography was carried out and revealed type A dissection (Stanford classification) from the ascending aorta to the right external iliac artery in the CT scan (Figure 2A,B). 
The aortic dissection also involved the brachiocephalic trunk, left common carotid artery, celiac trunk, superior mesenteric artery, and right renal artery (Figure 2C,D). The hemopericardium was also noted.
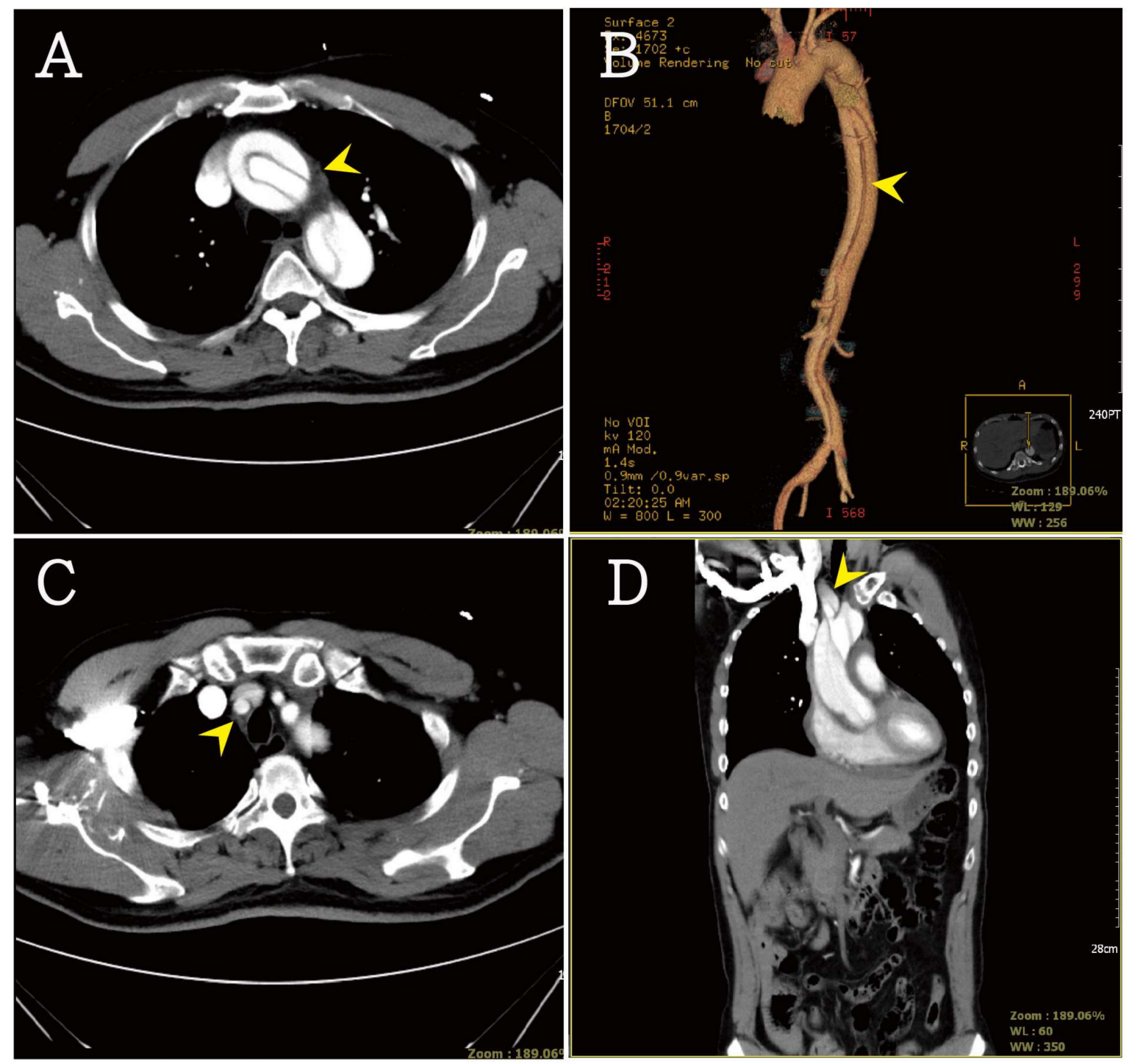

Figure 2. (A, B) Type A dissection (Stanford classification) with extension to the common iliac artery (arrow head) in the computed tomography (CT) scan. (C, D) The aortic dissection also involved the brachiocephalic trunk and the left common carotid artery (arrow head).

According to the above clinical symptoms and images, a type A dissection was suspected. An emergency ascending aorta replacement was arranged. However, during the surgery, a sudden onset of cardiac arrest was noted. Transesophageal echocardiography showed massive pericardic effusion. Resuscitative thoracotomies and open chest cardiac compressions were done. However, recurrent ventricular tachycardia and ventricular fibrillation were noted. After defibrillation and extracorporeal membrane oxygenation (ECMO) setup, the patient was transferred to ICU. Because of the poor outcome, the family asked to remove the patient from ECMO. The patient expired. A written informed consent was obtained from the patient. 


\section{Discussion}

Initial presentation with neurologic symptoms in aortic dissection patients has been rarely reported in previous studies and presents a frequency of about 37.7\% [2-4]. The predominant neurological symptoms can make it difficult for physicians to diagnose aortic dissection. Misdiagnosis or delayed diagnosis of aortic dissection may lead to catastrophic mortality and morbidity. In these patients, thrombolysis or thrombectomy, which are considered as the emergency stroke therapy, may be life-threatening in case of misdiagnosis of aortic dissection [5]. Regularly considering the possibility of aortic dissection is important for physicians to manage atypical stroke patients. Routine chest X-ray and physical examination are suggested as the first steps to include or exclude a diagnosis of aortic dissection, especially for patients presenting with hypotension, asymmetrical pulses, or heart murmur [6]. The initial neurological symptoms in type A aortic dissection were analyzed by Charly Gaul et al. [7], who described ischemic stroke (15.7\%), spinal cord ischemia (1\%), ischemic neuropathy $(10.8 \%)$, hypoxic encephalopathy $(2 \%)$, and syncope (5.9\%). The onset of the neurological symptoms may result from the extension of the dissection to aortic arch vessels, such as the brachiocephalic trunk and the left common carotid artery. The sudden onset of reduced cerebral perfusion and false lumen compressing the neurologic systems is also involved. In the study of Yuji Shono et al. [5], the involvement of supra-aortic artery and innominate artery was found in $100.0 \%$ of type A aortic dissection patients with CNS symptoms. The involvement of the common carotid artery and left subclavian artery was $63.6 \%$ and $36.4 \%$, respectively. The involvement of the supra-aortic artery and innominate artery was significant in patients presenting with neurological symptoms.

Hypertension is the most common presentation in acute stroke patients. In a nationally representative large data study, $69 \%$ of acute stroke patients had a systolic blood pressure (SBP) higher than $139 \mathrm{~mm} \mathrm{Hg}$ [8]. A similar result was reported in the study of J. D. Wallace et al. [9], which revealed that SBP higher than $150 \mathrm{~mm} \mathrm{Hg}$ was measured in $84 \%$ patients [9]. Compared to Yuji Shono et al. [5] analysis, the SBP of aortic dissection patients with neurological symptoms was $114.9 \pm 28.9 \mathrm{~mm} \mathrm{Hg}$ (mean $\pm \mathrm{SD}$ ), and the diastolic blood pressure was $66.5 \pm 17.3 \mathrm{~mm} \mathrm{Hg}$ (mean $\pm \mathrm{SD}$ ). No significant hypertension was reported. In our case, the initial blood pressure was $136 / 59 \mathrm{~mm} \mathrm{Hg}$, with no presentation of hypertension. In patients with neurological symptoms, the presentation of hypertension may be a sign pointing to a diagnosis of stroke.

Serum D-dimer, a fibrin degradation product, is a useful diagnostic tool for deep venous thrombosis, pulmonary embolism, and acute aortic dissection [10]. A cut-off value, reported for rule-out purposes, is $500 \mathrm{ng} / \mathrm{mL}$ that is similar to that indicated for pulmonary embolism and acute aortic dissection [10-12]. Using a cut-off value of $500 \mathrm{ng} / \mathrm{mL}$ for acute aortic dissection, the analysis revealed a sensitivity of $97 \%$, a negative predictive value of $96 \%$, a specificity of $56 \%$, a positive predictive value of a $60 \%$, positive likelihood ratio of 2.43 , and a negative likelihood ratio of $0.06[11,12]$. A concentration of serum D-dimer $<500 \mathrm{ng} / \mathrm{mL}$ is a sensitive screening tool for acute aortic dissection. However, in stroke patients, serum D-dimer is also increased. In 2002, Walter Ageno et al. [13] reported the serum D-dimer concentration in three major stroke subtypes: cardioembolic (mean \pm SEM: $2960 \pm 510 \mathrm{ng} / \mathrm{mL}$ ), atherothrombotic (mean \pm SEM: $1340 \pm 210 \mathrm{ng} / \mathrm{mL}$ ), and lacunar (mean \pm SEM: $670 \pm 80 \mathrm{ng} / \mathrm{mL}$ ). Serum D-dimer concentration cannot be used to differentiate the diagnosis of stroke and aortic dissection but is a useful rule-out tool for stroke and aortic dissection. Because of the above reasons, we did not measure serum D-dimer concentration in our patient. In our case, we highlighted the clinical features of acute aortic dissection which may be present together with neurological symptoms mimicking stroke and intracranial hemorrhage. We believe that the increased level of serum D-dimer may be significantly different in stroke and aortic dissection patients. This hypothesis needs to be confirmed by further studies. Although no known parameter is useful to provide strong evidence to distinguish these two diseases, a close follow-up of new symptoms, continuous monitoring of vital signs, early image studies, and timely medical and surgical interventions may improve patients' outcome. 
Author Contributions: Meng-Yu Wu and Ying-Wei Tsai wrote the paper; Ling-Chi Lee contributed to the organization of the figures; Yung-Chang Chien and Yu-Long Chen provided conceptual input; Giou-Teng Yiang, and Yueh-Tseng Hou proofread and organized the manuscript. All authors reviewed the final version of the manuscript.

Conflicts of Interest: The authors declare no conflict of interest.

\section{References}

1. Howard, D.P.J.; Sideso, E.; Handa, A.; Rothwell, P.M. Incidence, Risk Factors, Outcome and Projected Future Burden of Acute Aortic Dissection. Ann. Cardiothorac. Surg. 2014, 3, 278-284. [PubMed]

2. Lee, S.-J.; Kim, J.-H.; Na, C.-Y.; Oh, S.-S.; Kim, Y.-M.; Lee, C.-K.; Lim, D.-S. Eleven Years of Experience with the Neurologic Complications in Korean Patients with Acute Aortic Dissection: A Retrospective Study. BMC Neurol. 2013, 13, 46. [CrossRef] [PubMed]

3. Meszaros, I.; Morocz, J.; Szlavi, J.; Schmidt, J.; Tornoci, L.; Nagy, L.; Szep, L. Epidemiology and Clinicopathology of Aortic Dissection. Chest 2000, 117, 1271-1278. [CrossRef] [PubMed]

4. Blanco, M.; Diez-Tejedor, E.; Larrea, J.L.; Ramirez, U. Neurologic Complications of Type I Aortic Dissection. Acta Neurol. Scand. 1999, 99, 232-235. [CrossRef] [PubMed]

5. Shono, Y.; Akahoshi, T.; Mezuki, S.; Momii, K.; Kaku, N.; Maki, J.; Tokuda, K.; Ago, T.; Kitazono, T.; Maehara, Y. Clinical Characteristics of Type A Acute Aortic Dissection with CNS Symptom. Am. J. Emerg. Med. 2017, 35, 1836-1838. [CrossRef] [PubMed]

6. Gaul, C.; Dietrich, W.; Erbguth, F.J. Neurological Symptoms in Aortic Dissection: A Challenge for Neurologists. Cerebrovasc. Dis. 2008, 26, 1-8. [CrossRef] [PubMed]

7. Gaul, C.; Dietrich, W.; Friedrich, I.; Sirch, J.; Erbguth, F.J. Neurological Symptoms in Type A Aortic Dissections. Stroke 2007, 38, 292-297. [CrossRef] [PubMed]

8. Qureshi, A.I.; Ezzeddine, M.A.; Nasar, A.; Suri, M.F.; Kirmani, J.F.; Hussein, H.M.; Divani, A.A.; Reddi, A.S. Prevalence of Elevated Blood Pressure in 563,704 Adult Patients with Stroke Presenting to the ED in the United States. Am. J. Emerg. Med. 2007, 25, 32-38. [CrossRef] [PubMed]

9. Wallace, J.D.; Levy, L.L. Blood Pressure after Stroke. JAMA 1981, 246, 2177-2180. [CrossRef] [PubMed]

10. Perrier, A.; Desmarais, S.; Goehring, C.; de Moerloose, P.; Morabia, A.; Unger, P.F.; Slosman, D.; Junod, A.; Bounameaux, H. D-Dimer Testing for Suspected Pulmonary Embolism in Outpatients. Am. J. Respir. Crit. Care Med. 1997, 156 Pt 1, 492-496. [CrossRef] [PubMed]

11. Shimony, A.; Filion, K.B.; Mottillo, S.; Dourian, T.; Eisenberg, M.J. Meta-Analysis of Usefulness of D-Dimer to Diagnose Acute Aortic Dissection. Am. J. Cardiol. 2011, 107, 1227-1234. [CrossRef] [PubMed]

12. Marill, K.A. Serum D-Dimer Is a Sensitive Test for the Detection of Acute Aortic Dissection: A Pooled Meta-Analysis. J. Emerg. Med. 2008, 34, 367-376. [CrossRef] [PubMed]

13. Ageno, W.; Finazzi, S.; Steidl, L.; Biotti, M.G.; Mera, V.; d'Eril, G.M.; Venco, A. Plasma Measurement of D-Dimer Levels for the Early Diagnosis of Ischemic Stroke Subtypes. Arch. Intern. Med. 2002, 162, 2589-2593. [CrossRef] [PubMed] 